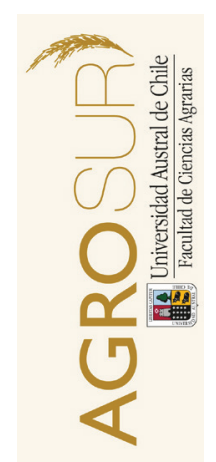

\title{
Valoración nutricional y productiva de diferentes granos de cereales germinados
}

\section{Nutritional and productive valuation of different germinated cereals}

\author{
Apráez, E. ${ }^{a}$, Calderón, D. ${ }^{b}$, Guerrero, L. ${ }^{b *}$ \\ ${ }^{a}$ Departamento de Producción y Procesamiento Animal, Universidad de Nariño. \\ Calle 18 Cr 50 Ciudadela Universitaria Torobajo, Pasto, Nariño, Colombia. \\ ${ }^{b}$ Estudiante de Zootecnia, Universidad de Nariño. Calle 18 Cr 50 Ciudadela Universitaria Torobajo, \\ Pasto, Nariño, Colombia.
}

\begin{tabular}{l} 
A R T I C L E I N F O \\
\hline Article history: \\
Received 02.06.2017 \\
Accepted 13.03.2018 \\
\hline Keywords: \\
Energy \\
Latency \\
Protein \\
Yield \\
\hline Original Research Article, \\
Animal Science
\end{tabular}

*Corresponding author:

Edmundo Apráez

\begin{abstract}
A B S T R A C T
The biomass yield, nutritional composition and content of secondary metabolites in grain and germinated cereals were evaluated. The treatments were: T1 yellow maize; T2 white corn; T3 capio corn; Barley T4; T5 galeras wheat; 66 sumatambo wheat and $\mathrm{T} 7$ oat. The yield of biomass, protein and energy was obtained by weighing the grain before and after the germination, as well as the content of phenols, saponins, alkaloids and steroids. Analysis of variance and comparison of means were performed using Duncan's test. The results obtained for biomass production indicated differences $(\mathrm{p}<0.05)$. T7 presented the highest fresh yield with $236.34 \%$, and the values for the remaining treatments ranged between 170.80 and $218.65 \%$. On dry basis T7 presented the highest $(\mathrm{p}<0.05)$ yield with $119.86 \%$, while the lowest was registered in T4 with $70.6 \%$. There were differences $(\mathrm{p}<0.05)$ recorded for proteins. In fresh germinated grains this component varied between $4.29 \%$ and $5.72 \%$, on a dry basis it fluctuated between $9.79 \%$ and $13.4 \%$. In the grain, the values ranged from $6.96 \%$ to $9.14 \%$ indicating an increase in germination with respect to the dry grain that had values ranging from 8 to $10.5 \%$. A higher protein yield was obtained in fresh germination of T3, while it was lower in T4. There were differences $(\mathrm{p}<0.05)$ in the energy content of germinated grains, obtaining $81 \%$ in T7 to $95 \%$ of NDT in T2 and T3. The values in grains ranged from $78 \%$ for T4 to $85 \%$ of NDT on T7. Positive energy yields were observed in all the treatments, except for $\mathrm{T} 4$.
\end{abstract}

\section{RESUMEN}

Se evaluó el rendimiento de biomasa, la composición nutricional y el contenido de metabolitos secundarios en cereales en grano y germinados. Los tratamientos fueron: T1 maíz amarillo; T2 maíz blanco; T3 maíz capio; T4 cebada; T5 trigo galeras; T6 trigo sumatambo y T7 avena. El rendimiento de biomasa, proteína y energía se obtuvo pesando el grano antes y después de la germinación, al igual que el contenido de fenoles, saponinas, alcaloides y esteroides. Se realizó un análisis de varianza y comparación de medias con la prueba de Duncan. Los resultados obtenidos para la producción de biomasa indicaron diferencias ( $\mathrm{p}<0,05)$, donde $\mathrm{T} 7$ presentó el mayor rendimiento en fresco con 236,34\%. Los restantes tratamientos tuvieron valores entre $170,80$ a $218,65 \%$. En base seca T7 presentó el mayor ( $p<0,05)$ rendimiento con 119,86\% y T4 fue el menor con 70,6\%. La proteína reportó diferencias ( $\mathrm{p}<0,05)$. En el germinado fresco la proteína varió entre 4,29\% y 5,72\% y en seco fluctuó entre $9,79 \%$ y $13,4 \%$. En el grano, los valores oscilaron entre 6,96\% y 9,14\%, indicando un incremento en el germinado respecto al grano seco, cuyos valores variaron entre 8 y 10,5\%. Se obtuvo mayor rendimiento proteico en el germinado fresco de T3 y menor en T4. En los germinados hubo diferencias ( $p<0,05)$ en energía, obteniéndose $81 \%$ en T7 a $95 \%$ de NDT en T2 y T3. En los granos los valores oscilaron entre 78\% de T4 a 85\% NDT de T7. En todos los tratamientos se observaron rendimientos energéticos positivos, salvo en T4.

Palabras clave: energía, latencia, proteína, rendimiento.

\section{INTRODUCCIÓN}

"En muchos países del mundo el consumo de germinados es muy común; en otros no son tan frecuentes ni se conocen sus propiedades, y en algunos se están conociendo recientemente; sin embargo, la germinación como fuente de alimentos, es uno de los procesos más antiguos usado desde hace siglos" (Ponce de León et al., 2013).

Históricamente, los cereales que normalmente crecen en espiga o mazorca han sido base alimentaria de varias civilizaciones. Son alimentos muy nutritivos, ricos en carbohidratos, fibra, minerales y proteína de buena calidad biológica. Entre las vitaminas de mayor 
concentración, se encuentran las del complejo B y están ubicadas en su germen. El refinamiento les hace perder su poder nutritivo, convirtiéndolas en harinas y féculas desnaturalizadas. Los cereales enteros, tostados, germinados o molidos, constituyen la base de una alimentación natural y son, quizás, el grupo de alimentos más importantes. Los cereales más reconocidos son: avena, centeno, arroz, millo, maíz y trigo en sus distintas variedades (FENALCE, 2015).

Cuando las semillas germinan, los carbohidratos, proteínas y aceites se vuelven aminoácidos predigeridos y azúcares naturales, de los que el embrión de la planta se alimenta a medida que crece. Cuando se usan en la alimentación animal, suministra la energía que es capaz de regular la temperatura corporal y mantener las funciones vitales de crecimiento, actividad, producción y reproducción. El proceso de germinación de granos, aparte de cosechar materiales frescos de buena calidad y sanos, contribuye a un plan de manejo ecológico con un mínimo impacto ambiental, sin el empleo de agroquímicos (Carballo, 2000).

La tendencia actual de buscar nuevas alternativas de alimentación y suplementación encaminada a reducir la utilización de alimentos concentrados, ha conllevado al uso de múltiples fuentes alimenticias, en especial de origen vegetal, por considerarse materias primas de bajo costo y de aceptable valor nutricional. Como una alternativa importante, se gesta la producción de germinados, tecnología de producción de biomasa obtenida a partir del crecimiento inicial de las plantas en los estados de germinación y crecimiento temprano de plántulas a partir de semillas viables (FAO, 2001).

Se ha incluido granos germinados en la alimentación de algunas especies menores como cuyes, conejos, aves, entre otros. Burbano y Lucero (2006) mencionan que los germinados de trigo y cebada constituyen recursos alimenticios de adecuado valor nutricional para conejos en la fase de levante y engorde, que permiten minimizar las pérdidas de rendimiento productivo ocasionadas por la escasez de alimentos tradicionales. Carballo (2000) menciona que el germinado en aves domésticas se ha usado desde 1929, especialmente el germinado de trigo para aumentar la producción de huevos.

Sosa y Cabrera (2009) reportan que con la inclusión de germinado en la dieta de los cerdos, la ganancia de peso fue superior, hecho que se puede atribuir a la mayor disponibilidad y solubilidad de los carbohidratos en el germinado, mejorando así la absorción de los mismos, contribuyendo a una mayor digestibilidad.

Jiménez y Noguera (2008) realizaron un estudio en cuyes en las fases de levante y ceba proporcionando a los animales en la dieta germinados de arveja, trigo y cebada, encontrando una mayor digestibilidad; resultados que se pueden explicar porque los componentes nutricionales sufren una transformación enzimática que permite un mejor aprovechamiento de éstos en el tracto digestivo.

Por lo anteriormente expuesto, se plantea que atributos productivos y de calidad nutricional de cereales germinados difieren entre sí. El objetivo de este estudio es valorar nutricional y productivamente granos germinados de maíz (Zea mays L.), cebada (Hordeum vulgare L.), avena (Avena sativa L.) y trigo (Triticum aestivum $\mathrm{L}$.).

\section{MATERIAL Y MÉTODOS}

El trabajo se desarrolló en la finca La Pradera, ubicada en "la vereda la Caldera", Municipio de Pasto, a una altura de $1.900 \mathrm{~m}$ s.n.m. y una temperatura media de $23{ }^{\circ} \mathrm{C}$; en la zona de vida de bosque húmedo Montano bajo (bh-MB)"(IGAC, 1972) .

\section{Instalaciones y equipos}

Se adecuó un invernadero para la producción de los germinados, con andamios en madera rústica en los cuales se acondicionaron entrepaños para colocar las bandejas plásticas de $55 \mathrm{~cm}$ de ancho por $77 \mathrm{~cm}$ de largo y 2,0 cm de profundidad, y un desnivel del $0,2 \%$. Los materiales se desinfectaron usando hipoclorito de sodio al $3,5 \%$.

\section{Prueba de germinación}

A los granos se les realizó pruebas de germinación para observar la calidad y la viabilidad de éstas. Para ello se tomaron 100 semillas de cada tratamiento, se envolvieron en papel periódico, se regaron con agua tres veces por día. Se revisó diariamente el brote del grano, se procedió a contar y obtener el porcentaje de germinación de la manera que se indica a continuación (FAO, 1983):

Germinación (\%) = $\left(\frac{\text { semillas germinadas }}{\text { número total de semillas en prueba }}\right) \times 100$

\section{Técnica de germinación}

El proceso de germinación se basó en el protocolo descrito por Caycedo et al. (2011), consistente en:

- Pesaje del grano libre de impurezas.

- Colocarlo dentro de un recipiente con agua.

- Dejar en remojo por un periodo de 24 horas.

- Escurrir el agua, posteriormente la siembra se efectuó en forma uniforme en cada bandeja con una cantidad de $500 \mathrm{~g}_{\text {bandeja }}{ }^{-1}$. Para ello se distribuyó una delgada capa de semillas, la cual no sobrepasó los 1,5 cm de altura o espesor. Luego de la siembra se taparon las semillas con papel 
periódico humedecido para facilitar la germinación de la semilla durante un periodo aproximado de ocho días.

- Regar agua tres veces por día, en una cantidad que permita humedecer el grano, sin provocar encharcamiento en las bandejas.

- Por último, se tomó una muestra de cada uno de los germinados, la cual se llevó al laboratorio para determinar los valores nutricionales y antinutricionales.

\section{Variables productivas}

\section{Rendimiento de biomasa (\%)}

Para valorar el rendimiento se pesó el grano antes y después del proceso de germinación (en fresco y en seco). El volumen de agua incorporada se determinó teniendo en cuenta el agua en el grano antes del proceso y en el grano germinado.

\section{Variables nutricionales y antinutricionales}

La determinación de materia seca se hizo por secado en estufa, la proteína se utilizó el método Kjeldahl; para ceniza y materia orgánica por incineración; en extracto etéreo por Soxhlet y la fibra cruda por Goering y Van Soest (1970). En los análisis de antinutricionales se utilizó las pruebas cuantitativas para determinar fenoles, saponinas, alcaloides y esteroides (AOAC, 2005).

\section{Energía}

El valor energético de los germinados se determinó por medio de la estimación de los nutrientes digestibles totales mediante la siguiente fórmula de Weiss (1993):

$$
E D\left(\text { Mcal kg MS }{ }^{-1}\right)=(6,149-0,178 \% \text { FDA })-0,02 \% \text { Hemicelulosa }+0,114 \% \text { Celulosa }
$$

Las fracciones de fibra detergente neutra y ácida, se determinaron de acuerdo con el método de Van Soest et al. (1991).

\section{Variables económicas}

Este análisis se realizó teniendo en cuenta los costos fijos (mano de obra) y los costos variables (costo forraje, semillas, medicamentos e insumos), para establecer la relación costo/beneficio, y determinar los costos de producción por kilogramo de germinado producido. Se tuvo en cuenta la siguiente ecuación:

$$
\text { Costo total }=\text { Costo fijo }+ \text { Costo variable }
$$

\section{Diseño experimental}

Para la producción de los granos germinados se aplicó un diseño completamente al azar con 7 tratamientos y 3 réplicas (tres bandejas por réplica), se realizó una prueba de homogeneidad de las varianzas. Los resultados obtenidos se procesaron en el paquete estadístico INFOSTAT, y la prueba de comparación Duncan porque se disponía de suficiente número de medias.

El modelo estadístico correspondiente fue:

$$
Y_{i j}=\mu+\tau_{i}+\varepsilon_{i j}
$$

Donde:

$Y_{i j}=$ Variable respuesta en el tratamiento $i$

$\mu=$ Media general del experimento

$\tau_{i}=$ Efecto del tratamiento $i$

$\varepsilon_{i j}=$ Error aleatorio.
La distribución de los tratamientos se realizó de la siguiente forma:

Cuadro 1. Distribución de los tratamientos.

Table 1. Distribution of treatments.

\begin{tabular}{cl}
\hline Tratamientos & Germinado \\
\hline 1 & Maíz amarillo (Z. mayz) \\
2 & Maíz blanco (Z. mayz) \\
3 & Maíz capio (Z. mayz) \\
4 & Cebada (H. vulgare) \\
5 & Trigo Galeras (T. aestivum) \\
6 & Trigo Sumatambo (T. aestivum) \\
7 & Avena (A. sativa) \\
\hline
\end{tabular}




\section{RESULTADOS Y DISCUSIÓN}

En el Cuadro 2 se presentan los resultados correspondientes a rendimiento de biomasa en los cereales germinados.

El contenido porcentual de materia seca de todos los cereales no tuvo variación ( $p>0,05$ ) (Cuadro 1), los granos germinados sí la presentaron $(\mathrm{p}<0,05)$, mostrando mayor contenido el tratamiento 3 (T3), correspondiente a maíz capio, con $58,39 \%$ y el menor contenido se observó en la cebada (T4) cuyo valor fue $32 \%$. Las variaciones observadas en el contenido de agua de los germinados podría estar asociada al tipo y cantidad de amilosa o amilopectina presentes en el endospermo de los granos, ya que tanto entre variedades y más aún entre especies, existen diferencias notables en la constitución de los almidones que integran esta fracción de los cereales. Al respecto, Karlsson et al. (1983) menciona que, en el endospermo, el almidón está presente como gránulos intracelulares de diferentes tamaños y depende de las especies de cereales. En el gránulo están presentes estructuras parcialmente cristalinas. El grado de la cristalinidad varía de 20 a $40 \%$ y es característica principal de la estructura de la amilopectina, polímero que le confiere menor solubilidad al almidón contenido en los granos y que, a su vez, estuvo ligado a la velocidad de germinación de los cereales, puesto que en las variedades de maíz y trigo el desarrollo de la radícula fue más tardío.

Se notó una alta influencia del tipo de cubierta o pericarpio que presentan los granos de cereales sobre la absorción de agua, esto debido a la estructura fibrosa de los mismos. En el caso del maíz, Singh (2009) afirma que las células del pericarpio son tubos huecos, que sirven como canales para la absorción de agua. Por el contrario, en el grano de cebada hay capas fibrosas que están cubriendo completamente el grano y la cáscara, está estrechamente unida a la capa de la semilla
(McKevith, 2004). Además, esta cascarilla protege al grano maduro del ataque de insectos y se elimina antes del procesamiento del grano (Collar, 2007).

Respecto al rendimiento porcentual en biomasa fresca, la avena presentó el valor más elevado, con 236,34\% ( $p<0,05)$, en comparación al T2 (maíz blanco), cuyo rendimiento fue el menor, de 170,8\% ( $p<0,05)$. Todos los tratamientos mostraron diferencias $(p<0,05)$ entre ellos, denotando que existe una disparidad en función de la variedad y especie de granos de cereales, denotando que cada especie tiene una capacidad higroscópica diferente en función del tipo de almidón que constituye cada especie. Carballo (2000) indica, que durante la fase de absorción de agua se inicia la actividad vital de la semilla, es decir, se reanuda el metabolismo, para lo cual se necesitan condiciones adecuadas de humedad, temperatura y oxígeno. Una vez reunidos estos factores, la semilla va aumentando de volumen por la absorción del agua, el embrión se hincha, se reblandecen las cubiertas protectoras y las reservas alimenticias principian una serie de reacciones químicas y biológicas que hacen que el embrión se desarrolle.

En el Cuadro 3, se presentan los resultados del rendimiento proteico en los cereales versus germinados. El análisis de varianza reveló diferencias $(\mathrm{p}<0,05)$ entre los tratamientos.

El contenido de proteína presentado en el grano de maíz blanco fue de $9,14 \%$, mayor ( $p<0,05)$ a las demás variedades evaluadas, cuyos valores oscilaron entre 6,96\% y 8,63\%. En relación con lo anterior, Méndez et al. (2005) mencionan que, en las variedades de maíz, el contenido de proteína puede oscilar entre $8 \%$ y $11 \%$ del peso del grano y, en su mayor parte, se encuentra en el endospermo. La UNAM (2013) indica que la cantidad de proteína difiere notablemente en los distintos cereales e inclusive dentro del mismo cereal de unas cosechas a otras; esto debido a la fuerte interacción

Cuadro 2. Rendimiento de biomasa en los cereales germinados.

Table 2. Biomass yield in germinated cereals.

\begin{tabular}{ccccc}
\hline Tratamientos & MS Gr & MS Gm & Rto BS Gm & Rto BF Gm \\
\hline 1 & $87,00 \pm 2,62 \mathrm{a}$ & $40,60 \pm 0,68 \mathrm{~d}$ & $101,01 \pm 1,02 \mathrm{c}$ & $216,45 \pm 1,90 \mathrm{~b}$ \\
2 & $87,00 \pm 2,93 \mathrm{a}$ & $50,20 \pm 0,77 \mathrm{~b}$ & $98,580 \pm 1,14 \mathrm{c}$ & $170,80 \pm 2,13 \mathrm{f}$ \\
3 & $87,00 \pm 2,93 \mathrm{a}$ & $58,39 \pm 0,77 \mathrm{a}$ & $119,50 \pm 1,14 \mathrm{a}$ & $178,06 \pm 2,13 \mathrm{e}$ \\
4 & $89,20 \pm 2,62 \mathrm{a}$ & $32,00 \pm 0,68 \mathrm{e}$ & $70,600 \pm 1,02 \mathrm{~d}$ & $196,36 \pm 1,90 \mathrm{~d}$ \\
5 & $89,25 \pm 2,93 \mathrm{a}$ & $44,30 \pm 0,77 \mathrm{c}$ & $101,84 \pm 1,14 \mathrm{c}$ & $204,59 \pm 2,13 \mathrm{c}$ \\
6 & $89,20 \pm 2,62 \mathrm{a}$ & $45,85 \pm 0,68 \mathrm{c}$ & $112,64 \pm 1,02 \mathrm{~b}$ & $218,65 \pm 1,90 \mathrm{~b}$ \\
7 & $87,00 \pm 2,62 \mathrm{a}$ & $43,97 \pm 0,68 \mathrm{c}$ & $119,86 \pm 1,02 \mathrm{a}$ & $236,34 \pm 1,90 \mathrm{a}$ \\
\hline
\end{tabular}

Letras diferentes en la misma columna indican diferencias estadísticas ( $\mathrm{p}<0,05)$. MS Gr (materia seca grano, \%); MS Gm (materia seca germinado, \%); Rto BS Gm (Rendimiento base seca germinado, \%); Rto BF Gm (Rendimiento base fresca germinado, \%). Desviación estándar ( \pm ). 
entre el genotipo y las condiciones ambientales que prevalecen durante el desarrollo y la maduración del grano. Los compuestos proteicos del grano se localizan en todos sus tejidos, pero el germen y la capa de aleurona concentran la mayor cantidad de compuestos nitrogenados.

De acuerdo con Martín et al. (2007), las proteínas mayoritarias presentes en los cereales son las prolaminas y glutelinas (60-80\% del total de proteínas), que constituyen la reserva que se sintetiza en las últimas etapas de maduración de la semilla y se moviliza durante la germinación, de modo que sirven de reserva de nitrógeno al germen. También contienen albúminas y globulinas (20-40\% del total), muchas de las cuales tienen actividad enzimática (amilasas, lipasas y proteasas), lo que es importante en el procesamiento de los cereales.

Debido a que los granos de cereales son ricos en nutrientes, los gérmenes de éstos son un componente valioso en la producción de alimentos funcionales (Ragaee et al., 2006; Sidhu et al., 2007). En este sentido, el germinado fresco de maíz capio que tuvo un mayor contenido proteico, de 5,72\% ( $p<0,05)$, y el de cebada un menor valor de $4,29 \%$. No obstante, los tenores proteicos del germinado resultaron inferiores a los del grano, esto debido a que el proceso de germinación favorece la incorporación de humedad, diluyendo la concentración de los nutrientes en proporciones variables. Al respecto Pita y Pérez (1998) sostienen que la hidratación de los tejidos de la semilla es un proceso físico con una duración variable según la especie. Hernández (2011) afirma que durante la fase de absorción del agua se inicia la actividad vital de la semilla, es decir, se reanuda el metabolismo, para ello se necesitan condiciones adecuadas de humedad, temperatura y oxígeno. Una vez reunidos estos factores, la semilla va aumentando de volumen por la absorción de agua, el embrión se hincha, se reblandecen las cubiertas protectoras y las reservas alimenticias principian una serie de reacciones químicas y biológicas que hacen que el embrión se desarrolle.

El germinado fresco de maíz capio (T3) presentó el mayor rendimiento proteico de 146,39\%, (p <0,05), respecto a los restantes germinados; la cebada tuvo la menor producción, con 97,53\%. Sin embargo, los cereales germinados restantes mostraron resultados positivos, obteniendo una ganancia en comparación con la proteína que presentan los granos.

Los resultados antes descritos pueden estar asociados a que al iniciarse la germinación de las semillas, previamente hidratadas, se produce la activación de la síntesis proteica y con ella se inicia la movilización de la reserva energética que es, fundamentalmente, el almidón. Marero et al. (1988) reportan un aumento de la calidad de proteína y micronutrientes durante la germinación de las semillas de cereales y leguminosas. Los germinados se consumen en fresco, lo que evita la alteración del valor nutricional por la cocción, como sucede con otros alimentos; además, son de fácil producción y de bajo costo. Éstos pueden contribuir en parte a resolver problemas de desnutrición y a corregir las carencias de la alimentación moderna a base de alimentos procesados (Domínguez de Diez, 1992).

Carballo (2000) reporta que: "La producción de granos germinados para uso forrajero, bajo control de temperatura, humedad relativa, densidad y buena calidad de la semilla, alcanza un rendimiento de 10-12 veces el peso de una semilla en forraje fresco y una altura de $20 \mathrm{~cm}$ aproximadamente en un periodo de 7-10 días". López y Ruales (2007) y Herrera et al. (2010) mencionan que es importante realizar la cosecha entre los 10 a 11 días después de la siembra, dado a que en este momento el forraje verde tendrá un mayor nivel de proteína, y por ende, un valor nutritivo superior que se traduce en mejores rendimientos productivos.

Cuadro 3. Rendimiento proteico en los cereales germinados.

Table 3. Protein yield in germinated cereals.

\begin{tabular}{ccccrc}
\hline Trat. & Prot G (BPS) & Prot G (BS) & Prot Gr (BF) & Prot Gr (BS) & Rto prot \\
\hline 1 & $7,83 \pm 0,09 \mathrm{c}$ & $9,00 \pm 0,10 \mathrm{c}$ & $4,59 \pm 0,02 \mathrm{~d}$ & $11,30 \pm 0,18 \mathrm{bc}$ & $126,82 \pm 1,15 \mathrm{c}$ \\
2 & $9,14 \pm 0,01 \mathrm{a}$ & $10,50 \pm 0,11 \mathrm{a}$ & $5,42 \pm 0,03 \mathrm{~b}$ & $10,80 \pm 0,20 \mathrm{c}$ & $101,39 \pm 1,28 \mathrm{f}$ \\
3 & $6,96 \pm 0,10 \mathrm{~d}$ & $8,00 \pm 0,11 \mathrm{~d}$ & $5,72 \pm 0,03 \mathrm{a}$ & $9,80 \pm 0,20 \mathrm{~d}$ & $146,39 \pm 1,28 \mathrm{a}$ \\
4 & $8,63 \pm 0,09 \mathrm{~b}$ & $9,70 \pm 0,10 \mathrm{~b}$ & $4,29 \pm 0,02 \mathrm{f}$ & $13,40 \pm 0,18 \mathrm{a}$ & $97,53 \pm 1,15 \mathrm{~g}$ \\
5 & $8,36 \pm 0,10 \mathrm{~b}$ & $9,39 \pm 0,10 \mathrm{~b}$ & $4,34 \pm 0,03 \mathrm{f}$ & $9,80 \pm 0,20 \mathrm{~d}$ & $106,18 \pm 1,28 \mathrm{e}$ \\
6 & $8,36 \pm 0,09 \mathrm{~b}$ & $9,39 \pm 0,10 \mathrm{~b}$ & $4,49 \pm 0,02 \mathrm{e}$ & $9,79 \pm 0,18 \mathrm{~d}$ & $117,44 \pm 1,15 \mathrm{~d}$ \\
7 & $8,41 \pm 0,09 \mathrm{~b}$ & $9,70 \pm 0,10 \mathrm{~b}$ & $5,06 \pm 0,02 \mathrm{c}$ & $11,50 \pm 0,18 \mathrm{~b}$ & $142,11 \pm 1,15 \mathrm{~b}$ \\
\hline
\end{tabular}

Letras diferentes en la misma columna indican diferencias estadísticas ( $<<0,05)$. Trat. (Tratamientos). Prot G (BPS) (proteína grano en base parcialmente seca, \%); Prot G (proteína grano base seca, \%); Prot Gr (BF) (proteína germinado base fresca, \%); Prot Gr (BF) (proteína germinado base seca, \%); Rto prot (rendimiento en proteína, \%). Desviación estándar ( \pm ). 
Los valores de proteína de los germinados en base seca, oscilaron entre $9,79 \%$ y $13,4 \%$, indicando un incremento respecto a los granos secos que fluctuaron en un rango de $8 \%$ a $10,5 \%$, por lo que resulta promisorio el proceso para mejorar también su aprovechamiento en el tracto gastrointestinal, incluso en animales monogástricos. Al respecto, NUTRIBIOTA (2017) manifiesta que durante la germinación la calidad de las proteínas se mejora gracias a la descomposición de las cadenas complejas de proteínas en aminoácidos libres y al aumento del contenido en aminoácidos esenciales (entre otros la lisina).

En el Cuadro 4 se presenta el rendimiento energético en los cereales versus germinados. El análisis de varianza reveló diferencias $(\mathrm{p}<0,05)$ entre los tratamientos.

Durante el proceso, el contenido energético en los germinados aumentó a un rango de $81 \%$ a $95 \%$ de $\mathrm{Nu}$ trientes Digestibles Totales (NDT), en comparación con la energía de los granos cuyos valores oscilaron entre $78 \%$ a $85 \%$ NDT $(\mathrm{p}<0,05)$; esto debido principalmente al contenido de almidón presente en el endospermo, el cual se transforma en el proceso de germinación en maltosa que es más digestible. Según FAO (2001), el endospermo que, en general, es la parte más grande del grano, constituye la fuente principal de energía, en la forma de un carbohidrato complejo, el almidón.

El germinado fresco de maíz capio (T3) mostró el mayor nivel energético, con 55\% de NDT, debido al mayor contenido de Extracto Libre de Nitrógeno (ELN). Al respecto, Luna y Narváez (2003) argumentan que: "el aumento de ELN puede influir positivamente en el contenido energético del alimento, especialmente carbohidratos solubles". En este sentido, Araneda (2015) menciona que el almidón es el principal carbohidrato de almacenamiento en los cereales, compuesto por dos homoglucanos insolubles en agua, amilosa y amilopectina.
En todos los tratamientos se presentaron rendimientos energéticos positivos, como consecuencia del alto contenido de carbohidratos solubles. Al respecto, Parsi et al. (2001) indican que el bajo contenido de fibra y la alta concentración de almidón hacen que el nivel de energía sea superior. En T4 el rendimiento fue menor, alcanzando $86 \%$ NDT, causado por el menor contenido de materia seca en comparación con los demás tratamientos.

En el Cuadro 5 se presentan los resultados de metabolitos secundarios, tanto de granos como germinados.

Los resultados obtenidos mostraron ausencia de metabolitos secundarios debido posiblemente, a que el proceso de germinación desencadena una actividad enzimática como mecanismo de defensa. Al respecto Valdés y Scull (2007) mencionan que son sustancias que se generan por el metabolismo secundario de las plantas para protegerse de depredadores como bacterias, hongos e insectos y otros animales. Pueden aparecer en cualquier parte de la planta incluyendo las raíces.

Sin embargo, hubo presencia de esteroles en poca cantidad en el grano de cebada y avena, en los demás cereales se observaron niveles altos.

En el Cuadro 6 se indica los costos (en pesos colombianos) calculados para cada tratamiento, para ello se tuvo en cuenta los costos fijos y variables.

El T1 presentó un menor costo, de $\$ 326,4 \mathrm{~kg}^{-1}$, esto debido a que el grano de maíz amarillo se consigue con mayor facilidad en el mercado y a un menor precio. Por su parte, el mayor costo lo presentó el T3 con un valor de $\$ 3160,1$, como consecuencia de la escasez del grano ya que éste es muy apetecido para el consumo humano. Los costos de los demás tratamientos oscilaron entre $\$ 471,9$ a $\$ 1027,5$. Este análisis permite inferir que los germinados de maíz, la cebada y el trigo resultan opciones económicamente viables de ser utilizadas en alimentación animal, no así la avena y el maíz capio.

Cuadro 4. Rendimiento energético en los cereales germinados.

Table 4. Energy efficiency in germinated cereals.

\begin{tabular}{cccccc}
\hline Trat. & NDT G (BPS) & NDT G (BS) & NDT Gr (BF) & NDT Gr (BS) & Rto NDT \\
\hline 1 & $73 \pm 1,50 \mathrm{a}$ & $84 \pm 1,11 \mathrm{ab}$ & $38,00 \pm 2,39 \mathrm{c}$ & $93,00 \pm 0,89 \mathrm{a}$ & $111,95 \pm 1,12 \mathrm{e}$ \\
2 & $70 \pm 1,67 \mathrm{a}$ & $81 \pm 1,24 \mathrm{bc}$ & $47,00 \pm 2,67 \mathrm{a}$ & $95,00^{\mathrm{a}} \pm 1,00 \mathrm{a}$ & $115,61 \pm 1,25 \mathrm{~d}$ \\
3 & $73 \pm 1,67 \mathrm{a}$ & $84 \pm 1,24 \mathrm{ab}$ & $55,00 \pm 2,67 \mathrm{a}$ & $95,00 \pm 1,00 \mathrm{a}$ & $135,96 \pm 1,25 \mathrm{a}$ \\
4 & $69 \pm 1,50 \mathrm{a}$ & $78 \pm 1,11 \mathrm{c}$ & $27,00 \pm 2,39 \mathrm{~d}$ & $86,00 \pm 0,89 \mathrm{~b}$ & $77,670 \pm 1,12 \mathrm{f}$ \\
5 & $70 \pm 1,67 \mathrm{a}$ & $79 \pm 1,24 \mathrm{c}$ & $40,75 \pm 2,67 \mathrm{bc}$ & $93,00 \pm 1,00 \mathrm{a}$ & $120,48 \pm 1,25 \mathrm{c}$ \\
6 & $70 \pm 1,50 \mathrm{a}$ & $79 \pm 1,11 \mathrm{c}$ & $41,20 \pm 2,39 \mathrm{bc}$ & $93,00 \pm 0,89 \mathrm{a}$ & $128,76 \pm 1,12 \mathrm{~b}$ \\
7 & $73 \pm 1,50 \mathrm{a}$ & $85 \pm 1,11 \mathrm{a}$ & $36,00 \pm 2,39 \mathrm{c}$ & $81,00 \pm 0,89 \mathrm{c}$ & $114,37 \pm 1,12 \mathrm{de}$ \\
\hline
\end{tabular}

Letras diferentes en la misma columna indican diferencias estadísticas $(\mathrm{p}<0,05)$. Trat. (Tratamiento). NDT G (BPS) (nutrientes digestibles totales en base parcialmente seca, \%); NDT G (BS) (nutrientes digestibles totales en base seca, \%); NDT Gr (BF) (nutrientes digestibles totales en el germinado base fresca, \%); NDT Gr (BF) (nutrientes digestibles totales en el germinado base seca, \%); Rto NDT (rendimiento en nutrientes digestibles totales, \%). Desviación estándar ( \pm ). 


\section{CONCLUSIONES}

Todos los cereales mostraron diferencias en producción de biomasa, denotando disparidad en función de la variedad y especie de grano. El mayor rendimiento se observó en el germinado de avena y el menor en la cebada.

Los valores proteicos en el grano fueron menores al germinado, indicando en todos los casos un incremento originado por el proceso. Hubo mayor rendimiento proteico en el germinado de maíz capio y menor en cebada.

El contenido energético aumentó después del proceso de germinación en todos los cereales, salvo en cebada.

Los germinados de maíz, la cebada y el trigo resultan opciones económicamente viables de ser utilizadas en alimentación animal, no así la avena y el maíz capio.

Cuadro 5. Análisis de metabolitos secundarios de diferentes variedades de granos de cereales.

Table 5. Analysis of secondary metabolites in different varieties of cereal grains.

\begin{tabular}{|c|c|c|c|c|c|}
\hline \multirow[t]{2}{*}{ Muestra } & \multirow[t]{2}{*}{ Tratamiento } & Saponinas & Fenoles & Esteroles & Alcaloides \\
\hline & & Espuma & Cloruro Férrico & Liebermann Burchard & Dragendorff \\
\hline \multirow{2}{*}{ Maíz amarillo } & Grano & - & - & +++ & - \\
\hline & Germinado & - & - & ++ & - \\
\hline \multirow{2}{*}{ Maíz blanco } & Grano & - & - & +++ & - \\
\hline & Germinado & - & - & ++ & - \\
\hline \multirow{2}{*}{ Maíz capio } & Grano & - & - & +++ & - \\
\hline & Germinado & - & - & ++ & - \\
\hline \multirow{2}{*}{ Cebada } & Grano & - & - & + & - \\
\hline & Germinado & - & - & + & - \\
\hline \multirow{2}{*}{ Trigo Galeras } & Grano & - & - & +++ & - \\
\hline & Germinado & - & - & + & - \\
\hline \multirow{2}{*}{ Trigo Sumatambo } & Grano & - & - & +++ & - \\
\hline & Germinado & - & - & + & - \\
\hline \multirow{2}{*}{ Avena } & Grano & - & - & + & - \\
\hline & Germinado & - & - & ++ & - \\
\hline
\end{tabular}

Bajo: +; Moderado: ++; Alto: +++; Negativo: -

Cuadro 6. Costo de cereales germinados por kilogramo.

Table 6. Cost of germinated cereals per kilogram.

\begin{tabular}{lcrrrrrr}
\hline Costos fijos (\$) & T1 & T2 & T3 & T4 & T5 & T6 & T7 \\
\hline Mano de obra (Horas día ${ }^{-1}$ ) & 4,0 & 4,0 & 4,0 & 4,0 & 4,0 & 4,0 & 4,0 \\
Transporte & 20,0 & 20,0 & 20,0 & 20,0 & 20,0 & 20,0 & 20,0 \\
Subtotal & $\mathbf{2 4 , 0}$ & $\mathbf{2 4 , 0}$ & $\mathbf{2 4 , 0}$ & $\mathbf{2 4 , 0}$ & $\mathbf{2 4 , 0}$ & $\mathbf{2 4 , 0}$ & $\mathbf{2 4 , 0}$ \\
Costos variables (\$) & & & & & & & \\
Grano & 680 & 760 & 5600 & 900 & 1200 & 1200 & 2400 \\
Insumos & 1,0 & 1,0 & 1,0 & 1,0 & 1,0 & 1,0 & 1,0 \\
Rendimiento & 2,20 & 1,71 & 1,78 & 1,96 & 2,04 & 2,18 & 2,36 \\
Subtotal & $\mathbf{6 8 1 , 0}$ & $\mathbf{7 6 1 , 0}$ & $\mathbf{5 6 0 1 , 0}$ & $\mathbf{9 0 1 , 0}$ & $\mathbf{1 2 0 1 , 0}$ & $\mathbf{1 2 0 1 , 0}$ & $\mathbf{2 4 0 1 , 0}$ \\
\cline { 1 - 5 } Costo germinado/Peso acumulado (\$) & $\mathbf{3 2 6 , 4}$ & $\mathbf{4 5 9 , 1}$ & $\mathbf{3 1 6 0 , 1}$ & $\mathbf{4 7 1 , 9}$ & $\mathbf{6 0 0 , 5}$ & $\mathbf{5 6 1 , 9}$ & $\mathbf{1 0 2 7 , 5}$ \\
\hline
\end{tabular}

T: Tratamientos. \$: peso colombiano. 


\section{REFERENCIAS}

Araneda, M., 2015. Hidratos de carbono (carbohidratos). Características y propiedades. Parte 1. http://www.edualimentaria.com/hidratos-de-carbono-caracteristicaspropiedades/parte1 (acceso, 07.06.2017).

Association of Official Analytical Chemists (AOAC), 2005. Official Methods of Analysis. Soil Chemistry, third ed. John Wiley \& Sons, Inc., New York.

Burbano, D., Lucero, R., 2006. Valoración nutritiva de cereales germinados de trigo (Triticum sp.), cebada (Hordeum vulgare) y maíz (Zea mays) en la alimentación de conejos (Orictolagus cuniculus). Tesis de grado de Zootecnia, Universidad de Nariño, Colombia. 79 p.

Carballo, C., 2000. Manual de procedimientos para germinar granos para alimentación animal. http://www.zoetecnocampo.com/Documentos/germinados.htm\#7 (acceso, 07.06.2017).

Caycedo, A., Zamora, A., Echeberry, S., Enríquez, R., Ortega, E., Burgos, M., Caycedo, M., 2011. Producción Sostenible de Cuyes. Alternativa económica para la conservación de cuencas hidrográficas en el Departamento de Nariño. Editorial Universitaria. Pasto, Colombia.

Collar, C., 2007. Cereales menores: Avena, sorgo, mijo, en: León, A., Rosell, C. (Eds.), De tales harinas tales panes. Granos, harinas y productos de panificación en Iberoamérica. Córdoba, Argentina, pp. 195-242.

Domínguez de Diez, G., 1992. Germinados: el alimento más perfecto y completo. 12 a ed. Editorial Posada, México.

Federación Nacional de Cultivadores de Cereales y Leguminosas (FENALCE), 2015. Cereales.http://www.fenalce. org/ fenalce/nueva/pg.php?pa=70\&d=Cereales $\% 20$ Fenalce. (acceso, 30.11. 2015).

Food and Agriculture Organization of the United Nations (FAO), 1983. Recolección, manipuleo, almacenaje y pretratamiento de las semillas de Prosopis en América Latina. Peter F. Ffolliott y John L. Thames Ed. Universidad de Arizona, Tucson, Arizona.

Food and Agriculture Organization of the United Nations (FAO), 2001. Manual técnico: Forraje verde hidropónico. Oficina Regional de la FAO para América Latina y el Caribe. Santiago, Chile.

Goering, H., Van Soest, P., 1970. Forage fiber analysis (Apparatus, reagents, procedures and some applications). Agricultural Research Service, United States Department of Agriculture. Agriculture Handbook $N^{\circ} 379$, Washington, D.C.

Hernández, E., 2011. Implementación de germinados en granja avícola de postura en San Salvador Tlanchinol. Técnico Superior Universitario Agrobiotecnología, Universidad Tecnológica de la Huasteca Hidalguense, Estado de Hidalgo, México, 9 p.

Herrera, E., Cerrillo, M., Juárez, A., Murillo, M., Ríos, F., Reyes, O., Bernal, H., 2010. Efecto del tiempo de cosecha sobre el valor proteico y energético del forraje verde hidropónico de trigo. Interciencia 35(4), 284-289. https://www. interciencia.net/wp-content/uploads/2018/01/284-cHERRERA-6.pdf

Instituto Geográfico Agustín Codazzi (IGAC), 1972. Dirección Agrológica. Breve descripción del sistema "Holdrige" para clasificar las zonas de vida o formaciones vegetales del mundo y su aplicación a Colombia. Bogotá, Colombia.
Jiménez, J., Noguera, M., 2008. Valoración Nutritiva de Granos Germinados de Arveja (Pisum sativa), Trigo (Triticum aestivum L.) y Avena (Avena sativa) en la alimentación de Cuyes tipo Carne (Cavia porcellus) durante la fase de levante y engorde. Tesis de grado de Zootecnia, Universidad de Nariño, Colombia. 92 p.

Karlsson, R., Olered, R., Eliasson A., 1983. Changes in starch granule size distribution and starch gelatinisation properties during development and maturation of wheat, barley and rye. Starch 35(10), 335-340. https://doi. org/10.1002/star.19830351002

López, D., Ruales, J., 2007. Evaluación de edad de cosecha y niveles de forraje verde hidropónico de cebada, maíz y trigo en el crecimiento de conejos de carne (Oryctolagus cuniculus) raza neozelandés. Tesis Ingeniero Agropecuario, Universidad Técnica del Norte, Ecuador. 127 p.

Luna, J., Narváez, J., 2003. Valoración nutritiva de los ensilajes de avena (Avena sativa) variedad Cayuse, L 15/85 y Obonuco, triticale 48 (Triticum SSR) en el levante de novillas Holstein mestizo. Tesis de grado de Zootecnia, Universidad de Nariño, Colombia. 56 p.

Marero, L., Payumo, E., Aguinldo, A., Homma, S., 1988. Nutritional characteristics of weaning foods prepared from germinated cereals and legumes. Journal of Food Science 53(5), 1399-1402. https://doi. org/10.1111/j.1365-2621.1988.tb09286.x

Martín, E., De Mateo, B., Miján, A., Pérez, A., Redondo, P., Sáenz, I., 2007. Pan y cereales. Editor Dirección General de Salud Pública y Alimentación, Madrid, España.

Méndez, G., Solorza J., Velázquez del Valle, M., Gómez, N., Paredes, O., Bello L., 2005. Composición química y caracterización calorimétrica de híbridos y variedades de maíz cultivadas en México. Agro Ciencia 39, 267-274.

McKevith, B., 2004. Nutritional aspects of cereals. Nutrition Bulletin 29(2), 111-142. https://doi.org/10.1111/ j.1467-3010.2004.00418.x

Nutrición Simbiótica Tradicional (NUTRIBIOTA), 2017. Los germinados de semillas y su cultivo casero. http:// www.nutribiota.net/blog/blog4.php/germinados-yfermentos-de-semillas?page $=4$. (acceso, 19.04.2017).

Parsi, J., Godio, L., Miazzo, R., Maffioli, R., Echevarría, A., Provensal, P., 2001. Valoración nutritiva de los alimentos y formulación de dietas. Curso de producción animal, Universidad Nacional de Río Cuarto, Provincia de Córdoba, Argentina.

Pita, J., Pérez, F., 1998. Germinación de semillas. Ministerio de agricultura pesca y alimentación, Madrid, España.

Ponce de León, C., Torija, M., Matallana, M., 2013. Utilidad en la alimentación de algunas semillas germinadas: brotes de soja y trigo. Boletín de la Real Sociedad Española de Historia Natural, Sección biológica 107(1-4), 47-55. http://historia.bio.ucm.es/rsehn/cont/publis/boletines/183.pdf

Ragaee, S., Abdel-Aal, E., Noaman, M., 2006. Antioxidant activity and nutrient composition of selected cereals for food use. Food Chemestry 98(1), 32-38. https://doi. org/10.1016/j.foodchem.2005.04.039

Sidhu, J., Kabir, Y., Huffman, F., 2007. Functional foods from cereal grains. International Journal of Food Properties 10(2), 231-244. https://doi. org/10.1080/10942910601045289 
Singh, V., 2009. Cereal Grains Structure \& Composition. 1st Brazil-U.S. Fulbright Biofuels Short Course. University of Illinois at Urbana-Champaign. July 27 - August 7, 2009. Sao Paulo, Brazil. http://www.iea.usp.br/midiateca/ apresentacao/singhbiofuels2.pdf

Sosa, J., Cabrera, G., 2009. Evaluación de diferentes niveles de inclusión de germinado de maíz amarillo (Zea mays) en la alimentación de cerdos en levante y ceba. Tesis de grado de Zootecnia. Universidad de Nariño, Colombia. $74 \mathrm{p}$.

Universidad Nacional Autónoma de México (UNAM), 2013. Composición Química de los Cereales-Proteínas. http://olimpia.cuautitlan2.unam.mx/semillas/index. php?option=com_content\&view=article\&id=16\&Itemid $=20 \&$ limitstart $=2$ (acceso, 07.06.2017).
Valdés, S., Scull, I., 2007. Factores antinutricionales en recursos alimentarios tropicales para especies monogástricas. IX Encuentro de Nutrición y Producción en Animales Monogástricos. Universidad de La República, Noviembre de 2007, Montevideo, Uruguay, pp. 93-98. http://anatomiayplastinacion.wikispaces.com/file/ view/IX+encuentro+de+nutricion.pdf

Van Soest, P., Robertson, J., Lewis, B., 1991. Methods for dietary fiber, neutral detergent fiber and nonstarch polysaccharides in relation to animal nutrition. Journal of Dairy Science 74(10), 3583-3597. https://doi.org/10.3168/ jds.S0022-0302(91)78551-2

Weiss, W.P., 1993. Predicting energy values of feeds. Journal of Dairy Science 76(6), 1802-1811. https://doi. org/10.3168/jds.S0022-0302(93)77512-8 
